\title{
Role of surface vibrational properties on cooperative phenomena in spin-crossover nanomaterials
}

\author{
Mirko Mikolasek, ${ }^{1,2}$ Gautier Félix, ${ }^{1}$ Gábor Molnár, ${ }^{1}$ Férial Terki, ${ }^{2}$ William Nicolazzi, ${ }^{1,}{ }^{*}$ and Azzedine Bousseksou ${ }^{1, \dagger}$ \\ ${ }^{1}$ Laboratoire de Chimie de Coordination, CNRS UPR-8241 and Université de Toulouse, UPS, INP, Toulouse France \\ ${ }^{2}$ Institut Charles Gerhardt, Université Montpellier 2, place Eugène Bataillon, Montpellier France \\ (Received 6 June 2014; revised manuscript received 17 July 2014; published 4 August 2014)
}

\begin{abstract}
The influence of surface/interface on the lattice dynamics of spin crossover nanoparticles has been investigated by a spring-ball model solved by Monte Carlo methods. The bond cohesion energy of the model has been extracted from Mössbauer spectroscopy measurements performed on the model compound $\mathrm{Ni}_{3}\left[\mathrm{Fe}(\mathrm{CN})_{6}\right]$. We show that the coupling between bulk and surface vibrational properties, which drastically affects the mechanical properties of the whole particle below a characteristic size, has a major impact on the phase stability of the particles. In the case of free surfaces, the Debye temperature decreases with the size and the first-order nature of the spin transition disappears. On the other hand, a hardening of the surface bonds leads to increasing particle stiffness with the size reduction. In this case, a persistence of the hysteretic behavior in the spin transition curve is also predicted in good agreement with previous theoretical and experimental results.
\end{abstract}

DOI: 10.1103/PhysRevB.90.075402

PACS number(s): 64.70.Nd, 64.60.De, 75.30.Wx

\section{INTRODUCTION}

In recent years, the synthesis, the design, and the characterization of spin crossover ( $\mathrm{SCO}$ ) nanoobjects have received a growing interest [1-3], due to their potential application in the elaboration of sensors, new generation of electronic/photonic nanodevices, and micro- or nanoactuators [4]. Indeed, SCO molecules are able to switch from a low-spin (LS) to a highspin (HS) state with the application of an external stimulus [5] (temperature, pressure [6], magnetic field [7], light irradiation [8], pulsed laser irradiation [9],...). In the solid state, the spin state change can be abrupt with a hysteresis phenomenon (first-order transition) whose existence is highly dependent on the nature of the elastic interactions (the electron-phonon coupling) in the crystal packing $[10,11]$.

At the nanometric scale, recent experimental observations in SCO nanoparticles have shown discordant results and have brought out new fundamental questions concerning the existence of the bistability [12]. While the loss of the hysteretic behavior has been observed in many cases with the size reduction (below approximately 50-100 nm) [13-16], an unexpected presence of the bistability phenomenon has been detected in some SCO nanoparticles smaller than $10 \mathrm{~nm}$ [17-21]. Size effects are generally attributed to the increase of surface-to-volume ratio implying a predominance of the surface physical properties and a higher sensitivity of nanoobjects to the surrounding external chemical environment [20]. The mechanical properties of the matrix as well as the physicochemical properties of the matrix-particle interface may play an important role in the observed properties, even for micrometric particles [22]. Most of the theoretical investigations using Ising-like models [23-27], spin-phonon Hamiltonians [28], or mechanoelastic models [29] predict an ineluctable loss of the memory effects below a critical size for free nanoparticles.

However, it is well known that various physico-chemical properties of a nanoobject can be size dependent [30], in

\footnotetext{
*willliam.nicolazzi@lcc-toulouse.fr

†azzedine.bousseksou@1cc-toulouse.fr
}

particular the elastic and vibrational properties. For example, the measure of the Debye temperature of a series of Prussian-blue complexes has highlighted a hardening of the materials for very small nanoparticles [31]. In this work, a core-shell thermodynamic approach has been employed to show that this size dependence of the vibrational properties can enhance the electron-phonon coupling and can lead to a reopening of the hysteresis loop at very low sizes. Moreover, a recent theoretical study combining statistical physics and nonextensive thermodynamics has shown that the surface energy of a free nanoparticle depends on the characteristics of the intermolecular forces of the whole particle [32]. The dependence of the surface energy with the stiffness of the particle core supposes that the shell and the core vibrational properties are strongly coupled. Reciprocally, surfaces can influence the physical properties of the inner particle and are able to play a key role in the modifications of elasticity with the size reduction and therefore in the SCO properties at the nanoscale.

In this paper, we propose to investigate the surface effects on the vibrational properties of nanoparticles through the numerical simulation of the size dependence of the Debye temperature using an original Monte Carlo method. In particular, the spatial range of the surface properties within the nanocrystal as well as the finite size effects on the SCO properties are discussed. The manuscript is organized as follows. Section II is devoted to the introduction of the model and the computational details of the Monte Carlo methods. The numerical extraction of cohesion energy from Mössbauer spectroscopy measurements is described in Sec. III as well as the consequence of the size dependence of vibrational properties on the SCO behavior. We finally conclude in Sec. IV.

\section{MODEL AND COMPUTATIONAL DETAILS}

\section{A. Spin-phonon Hamiltonian}

In the following, we describe the spin-phonon model introduced in a previous work, the so-called anharmonic Isinglike model [33]. Let us consider a cubic lattice constituted of $N$ molecules (or sites) whose vibronic states are described by a 
degenerate two-level model [34]. Considering that this model is completely equivalent to a nondegenerated Ising model under a temperature dependent field, the on-site Hamiltonian can be expressed as

$$
\mathcal{H}_{\mathrm{sp}}=\frac{1}{2}\left(\Delta-k_{B} T \ln \frac{g_{\mathrm{HS}}}{g_{\mathrm{LS}}}\right) \sum_{i=1}^{N} \sigma_{i},
$$

where $k_{B}$ and $T$ stand for the Boltzmann constant and the temperature, respectively. $\sigma_{i}= \pm 1$ are the eigenvalues of the fictitious spin operator, which represents the spin state of the $i^{\text {th }}$ molecule ( +1 for the HS state and -1 for the LS state). $\Delta$ represents the energy difference between the two molecular states, which favors the LS state at low temperatures, whereas the vibrational and electronic degeneracy ratio between the two spin states $g_{\mathrm{HS}} / g_{\mathrm{LS}}$ favors the HS state at high temperatures. In order to simulate the elastic nature of the interaction and the electron-phonon coupling, the lattice degrees of freedom are taken into account and coupled with the fictitious spin through a spin state dependent potential energy, corresponding to the electron-phonon interaction $[11,33]$ :

$$
\mathcal{H}_{\mathrm{el}}=\sum_{\langle i, j\rangle} A\left(\sigma_{i}, \sigma_{j}\right) V_{\mathrm{el}}\left(r_{\langle i j\rangle}, \sigma_{i}, \sigma_{j}\right),
$$

where

$$
V_{\mathrm{el}}\left(r_{\langle i j\rangle}, \sigma_{i}, \sigma_{j}\right)=\left(\frac{r_{\langle i j\rangle}^{0}\left(\sigma_{i}, \sigma_{j}\right)}{r_{\langle i j\rangle}}\right)^{6}-2\left(\frac{r_{\langle i j\rangle}^{0}\left(\sigma_{i}, \sigma_{j}\right)}{r_{\langle i j\rangle}}\right)^{3} .
$$

The sum $\langle i, j\rangle$ is restricted to the nearest neighbors (NN). $r_{\langle i j\rangle}$ and $r_{\langle i j\rangle}^{0}\left(\sigma_{i}, \sigma_{j}\right)$ correspond to the instantaneous and equilibrium distances between two $\mathrm{NN}$ sites, respectively. $V_{\mathrm{el}}$ is the two-body interaction Lennard Jones potential simulating the elastic stress exerted by neighboring molecules $j$ on a molecule $i$ [33]. The properties of the interaction Hamiltonian $\mathcal{H}_{\mathrm{el}}$ [cohesion energy $A\left(\sigma_{i}, \sigma_{j}\right)$ and the equilibrium distances $\left.r_{\langle i j\rangle}^{0}\left(\sigma_{i}, \sigma_{j}\right)\right]$ are dependent on the spin states of the molecules forming the bonds (HS-HS, LS-LS, LS-HS) and traduces the local elastic deformations generated by the switching of molecules in the crystal packing $[35,36]$. The next nearestneighbor (NNN) interactions are added in the simulation to the sole purpose of preserving the symmetry of the lattice [37]. The total Hamiltonian can be written as

$$
\mathcal{H}^{(1)}=\mathcal{H}_{\mathrm{sp}}+\mathcal{H}_{\mathrm{el}} \text {. }
$$

A first-order transition with a hysteresis cycle can be obtained either by increasing (i) the difference in bond strength $([A(1,1)-A(-1,-1)],[A(1,1)-A(-1,1)]$ and $[A(-1,-1)-A(-1,1)])$ between the two spin states or (ii) the stiffness of the lattice [38]. In this work, we limit the study to the size effects on the stiffness of the lattice and its consequences on the spin transition properties.

\section{B. Monte Carlo simulation}

In order to investigate the thermoelastic properties of the Hamiltonian (4), the estimations of thermal quantities are performed by Monte Carlo methods in the isothermal and isobaric ensemble $(T, P=0, N)$. We define the mean number of molecules in the HS state, the so-called HS fraction $n_{\mathrm{HS}}$, which is directly related to the thermal average fictitious spin $\langle\sigma\rangle$ :

$$
n_{\mathrm{HS}}=\frac{1+\langle\sigma\rangle}{2} .
$$

$n_{\mathrm{HS}}$ is the usual order parameter to follow the spin transition.

The calculation of elastic/vibrational properties can be achieved either by the numerical estimation of the bulk modulus or by the simulation of the Debye temperature $\theta_{D}$. While the former corresponds to an overall response of the system volume to the application of an isostatic pressure and is also related to spatially averaged thermal quantities, the latter can be deduced from the mean-square displacement of molecules $\left\langle u^{2}\right\rangle$ (vide infra):

$$
\left\langle u^{2}\right\rangle=\frac{1}{N} \sum_{1}^{N}\left\langle u_{i}^{2}\right\rangle,
$$

where $\left\langle u_{i}^{2}\right\rangle=\left\langle\left(r_{i}-\left\langle r_{i}^{0}\right\rangle\right)^{2}\right\rangle$ is the mean-square displacement of the $i$ th site. This local quantity allows to access to the spatial distribution of bond vibrations and its knowledge can also probe the impact of surface vibrations on the crystal stiffness.

The coordination defects at the surface (missing bonds due to the creation of surface) can be simply taken into account by applying free boundary conditions. Other boundary conditions can also be used to simulate the difference between the bulk and the surface in terms of chemical properties, either with a local modification of the ligand field $\Delta$ at the surface [32] or by fixing molecules at the surface in the HS state (specific boundary conditions) [24,28], which forms a core-shell system. These latter conditions explain in a simple manner the down-shift of the transition temperature and the existence of a residual HS fraction at low temperatures and are interpreted as a "negative pressure," which acts on the nanoparticle core. A physical origin of the size evolution of the transition temperature has been proposed through a core-shell thermodynamic model, which introduces a spin state dependent surface energy [31]. This interface energy can be developed in chemical and mechanical (matrix and local surface stresses...) terms, even if all these contributions are correlated. The resulting difference between the HS and the LS surface energies constitutes the driving force of the spin transition at the nanoscale [31]. In this present work, we are interested in the influence of a free interface on the lattice dynamics of nanoparticles, i.e., without additional constraints. The calculation of thermal quantities $n_{\mathrm{HS}}$ and $\left\langle u_{i}\right\rangle$ by Monte Carlo simulation is based on a sampling method of accessible microstates in the phase space using stochastic dynamics governed by a master equation formalism [33]. Spin state and position variables of each site are successively updated according to the usual Metropolis criterion [39]. A particular attention has been paid for the control and the update of the system volume due the existence of large fluctuations, especially for very small sizes. Indeed, the displacement of one site can imply a significant variation of the volume and large distortions of the cubic lattice. In addition, the numerical estimation of the local mean-square displacements has to be performed carefully for very small system sizes due to the open boundary conditions. Indeed, translations or rotations of the whole nanoparticle, which are usually neglected for massive 
systems due to their larger inertia, have to be considered here. These additional degrees of freedom prevent to define easily an equilibrium position for each site $i$. To solve this problem, three spatial reference axis are used: the center of mass of the lattice, corresponding to the averaged positions of all sites and two vectors along the crystallographic directions [100] and [010]. The center of mass defines the origin of the Cartesian coordinate system and the two vectors allow the control of the random rotations of the whole nanoparticle. Positions and rotations of the particle have been checked at every mean-square displacement measure and controlled with these three spatial references. In the following and if not mentioned otherwise in the text, thermal quantities have been estimated as the average over 1000 configurations obtained from independent MC simulations (ensemble averages).

\section{RESULTS AND DISCUSSIONS}

In a first step, we study the size evolution of elastic properties of a 3D cubic crystal, which does not diplay a phase transition in order to avoid the complications that may arise due to the presence of multiple (HS and LS) phases in various proportions. The Hamiltonian (4) is simplified as follows:

$$
\mathcal{H}_{\mathrm{el}}^{(2)}=\sum_{\langle i, j\rangle} \epsilon V_{\mathrm{el}}\left(r_{\langle i j\rangle}, r_{\langle i j\rangle}^{0}\right),
$$

where $\epsilon$ is the cohesion energy of the crystal.

\section{A. Extraction of bond cohesion energy from Mössbauer spectroscopy}

First, it is important to show that orders of magnitude of the different structural and elastic quantities contained in the Hamiltonian (7) can be extracted from experimental data. Indeed, the equilibrium position $r_{\langle i j\rangle}^{0}$ can be obtained from lattice parameters and atomic coordinates measured by diffractions techniques and the cohesion energy $\epsilon$ or the elastic constant can be estimated from 57-Fe Mössbauer spectroscopy, which allows the determination of the Debye temperature $\theta_{D}$ for iron-containing materials (which is the case for many SCO materials) by probing the local environment (via the mean-square displacement) of this atom.

The Debye temperature $\theta_{D}$ is extracted from the Mössbauer spectroscopy measurement, by a linear fit of the temperature dependence of the Mössbauer spectrum area $A$, using the wellknown high-temperature limit [40] :

$$
\ln (A) \propto \ln (f) \approx-\frac{6 E_{R}}{k_{B} \theta_{D}^{2}} T,
$$

where $f$ is the Lamb-Mössbauer factor, $k_{B}$ is the Boltzmann constant, and $E_{R}$ is the recoil kinetic energy of iron.

On the other hand, the Lamb-Mössbauer factor $f$ is directly related to the mean-square displacement:

$$
f=\exp \left(-\frac{k^{2}\left\langle u^{2}\right\rangle}{3}\right),
$$

where $\vec{k}$ is the wave vector of the incident photon beam. By injecting in Eq. (9) the MC calculation of the mean-square displacement $\left\langle u^{2}\right\rangle$ for a given cohesion energy $\epsilon$, a numerical Debye temperature $\theta_{D}(\epsilon)$ is deduced from Eq. (8) and can be compared with the experimental result $\theta_{D}^{\exp }$. Then, the cohesion energy $\epsilon$ corresponding to $\theta_{D}(\epsilon)=\theta_{D}^{\exp }$ is determined by reverse Monte Carlo simulation, which minimizes the difference between the numerical and experimental data [41].

In the following, we choose to extract the order of magnitude of the cohesion energy $\epsilon$ of Eq. (7) from the elastic properties of $\mathrm{Ni}_{3}\left[\mathrm{Fe}(\mathrm{CN})_{6}\right]$ studied by Mössbauer spectroscopy in a previous work [31]. This compound does not present a spin conversion, but its structure is very similar to those of spin transition coordination networks. We perform Monte Carlo simulation on a $N=30^{3}$ cubic system with an equilibrium position $r_{\langle i j\rangle}^{0}=5.1 \AA$, corresponding to the experimental $\mathrm{Fe}-\mathrm{Ni}$ distance. In the simulation, nickel atoms are assimilated to iron ones for convenience, keeping in mind that the 57-Fe Mössbauer spectroscopy is only sensitive to iron nuclei. Sites close to the surface have been disregarded in the calculation of the mean-square displacement because finite size effects are always present in simulating the system, while they are negligible in bulk materials. The disregarded surface sites are defined by a characteristic surface thickness (cf. Sec. III B) such as the mean-square displacement of core sites is close to the bulk material. Figure 1 depicts the thermal evolution of the Mössbauer spectrum area $A$ of $\mathrm{Ni}_{3}\left[\mathrm{Fe}(\mathrm{CN})_{6}\right]$ (red crosses) in a logarithmic representation. The experimental data give $\theta_{D}^{\exp }=187(9) \mathrm{K}$ [31]. The thermal evolution of the mean-square displacement calculated from MC simulation (Fig. 1, blue circles) shows the expected linear thermal dependence in the high-temperature limit in agreement with the classical treatment of phonons in the harmonic approximation. Anharmonicity effects due to the Lennard-Jones shape of the elastic potential, which arise close

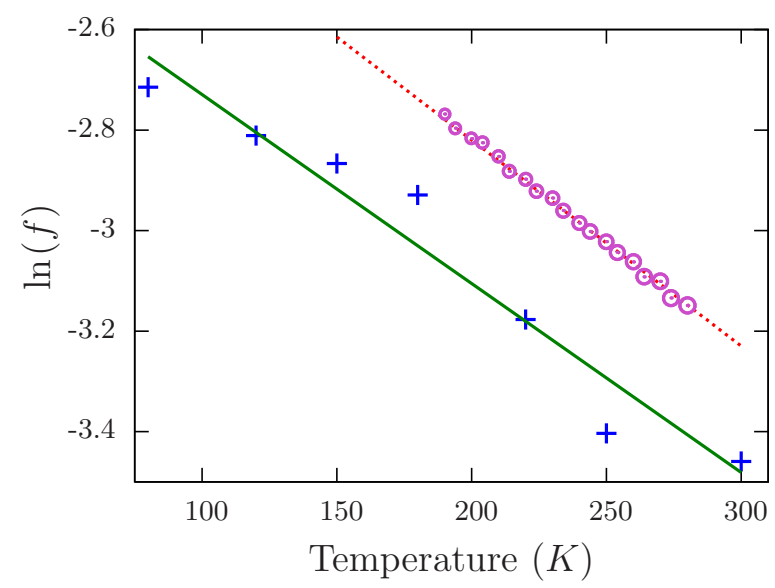

FIG. 1. (Color online) Experimental data (blue crosses) and numerical simulation (purple circles) of the thermal evolution of the Lamb-Mössbauer factor $f$ in a logarithmic representation. The straight green line corresponds to a linear fit of experimental data obtained on the compound $\mathrm{Ni}_{3}\left[\mathrm{Fe}(\mathrm{CN})_{6}\right]$ using the Debye model in the high-temperature limit (from Ref. [31]). MC simulation has been realized on a system with $N=30^{3}$ sites and the temperature sweep has been performed with a heating rate of $5 \times 10^{-4} \mathrm{~K} \mathrm{MCS}^{-1}$ (Monte Carlo step) in the warming and cooling modes, the initial system configuration being the final configuration of the previous temperature. The sizes of circles are directly related to the MC numerical errors. 
(a)

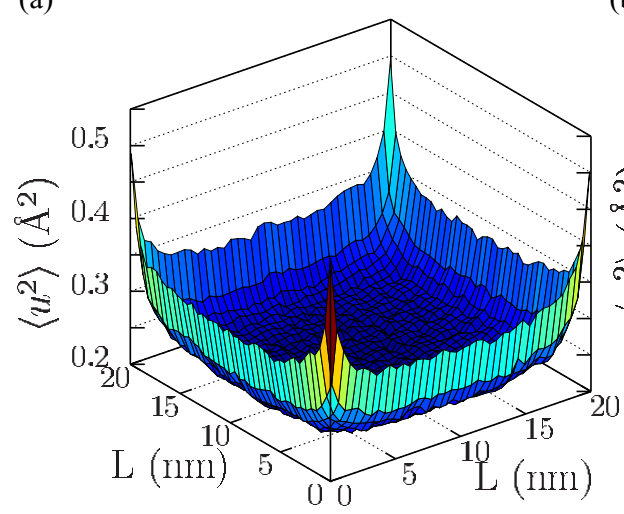

(b)

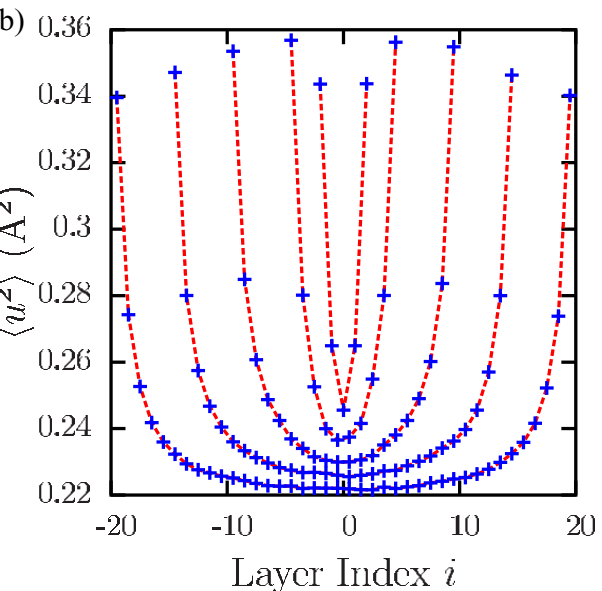

(c)

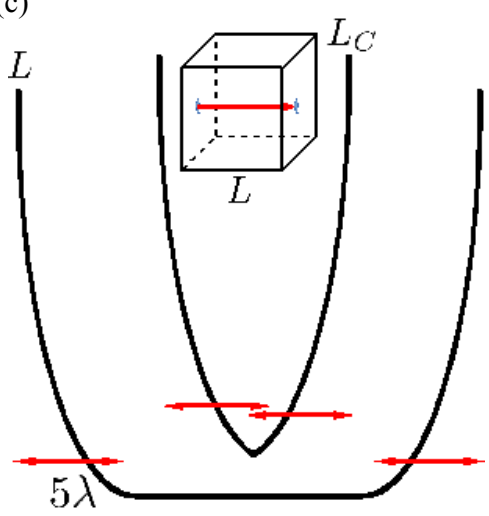

FIG. 2. (Color online) (a)Spatial distribution of the local mean-square displacements $\left\langle u_{i}^{2}\right\rangle$ for a section of the cubic system of size $N=40^{3}$ with free surfaces. (b) Corresponding averaged profile of $\left\langle u_{i}^{2}\right\rangle$ for different system sizes $\left(N=5^{3}, 10^{3}, 20^{3}, 30^{3}\right.$, and $\left.40^{3}\right)$. The center of the cube is taken as the reference to identify the layers between two opposite surfaces. For (a) and (b), MC simulations have been performed at $T=200 \mathrm{~K}$ with a cohesion energy $\epsilon=6000 \mathrm{~K}$. (c) Schematic representation of the surface effects on the lattice dynamics at the nanometric scale where $\lambda$ is the characteristic surface thickness. When $L \approx L_{c}$, the vibrational properties of the system are completely different from the corresponding bulk material $\left(L \gg L_{c}\right)$. (Insert) Schematic representation of the path along which the profile of $\left\langle u_{i}^{2}\right\rangle$ has been determined.

to the melting process, lead to a deviation from this linear dependence at higher temperatures (not shown here). After convergence of the reverse Monte Carlo algorithm, a slope of $\theta_{D}=186.7 \mathrm{~K}$ is numerically obtained, corresponding to a value of $\epsilon=26800(200) \mathrm{K} \approx 2.31(2) \mathrm{eV}$. This value is of the same order of magnitude than covalent bond energies, in good agreement with the coordination network structure of this material. Similar extractions of cohesion energy can be performed on molecular crystals, but it is important to point out that the extraction of the Debye temperature has to be carefully performed in this latter case, mainly due to the fact that Mössbauer spectroscopy techniques only probe the close environment of iron atoms. The estimation of the Debye temperature would be only valid for the iron sublattice. In molecular crystals, the weaker intermolecular interactions would lead to lower cohesion energies than coordination networks, typically around $\epsilon \approx 5000-6000 \mathrm{~K}$. This combined numerical/experimental method can be systematically applied to switchable SCO materials for which the cohesion energies of the HS and LS phases could be extracted. The knowledge of these two quantities as well as their size evolution is of paramount importance since the cooperative spin transition mechanisms are mainly governed by the difference of stiffness between the HS and LS phases and the intermolecular vibrational properties of the materials. This latter can be strongly affected when the size is decreased due to the presence of surfaces.

\section{B. Surface effects}

The physicochemical properties of a material are expected to change when the system size decreases below a "critical size" [16]. Generally, this "critical size" may be different according to the physical phenomena and the size dependence of physicochemical properties involved in, making it difficult to give a clear definition. The surface/interface physical properties are different from the inner particle due to coordination defects, surface reconstructions or/and external environments, which hinder or enhance the observed physical phenomenon. In SCO materials, a possible origin of the disappearance or the survivance of the memory effect (first-order transition) probably comes from a modification of the elastic behavior induced by the growing influence of the surface vibrational properties.

This change in the lattice dynamics with the size can be numerically studied by inspecting the spatial distribution of the local mean-square displacement $\left\langle u_{i}^{2}\right\rangle$. Figure 2(a) shows an example of a mapping of $\left\langle u_{i}^{2}\right\rangle$ for a section of the cubic lattice. The local mean-square displacement is inhomogeneously distributed and is higher at the surfaces, especially at the corner of the section where the coordination number is the lowest. This can be understood by the fact that missing bonds at the surfaces allow a higher freedom of movement than in the core. It is important to note that the mean-square displacement decreases continuously and progressively as one moves away from the surfaces. Surface effects penetrate deep into the material and impact the vibrational properties of the first layers of molecules close to the side or the corner of the system. Beyond a finite distance from surfaces, $\left\langle u_{i}^{2}\right\rangle$ reaches a threshold value corresponding to the mean-square displacement of the bulk.

The size evolution of the mean profile of the local mean-square displacement $\left\langle u_{i}\right\rangle$ calculated between two points located at the center of two opposite faces of the cube is represented in Fig. 2(b). A characteristic value of the surface thickness, noted $\lambda$, can be estimated from an exponential fit of the different profiles (see Appendix A). From this characteristic value, a critical size $L_{c}$ can be deduced. $L_{c} / r_{\langle i j\rangle}^{0}$ is not significantly dependent either on the cohesive energy $\left[A\left(\sigma_{i}, \sigma_{j}\right)\right]$ or on the lattice parameter but can be weakly influenced by the temperature and the lattice structure. When the size of the system $L$ is much greater than $L_{c}$, surface effects can be ignored and the thermodynamic limit can be considered as valid. However, when $L \sim L_{c}=7-8 \mathrm{~nm}$, 


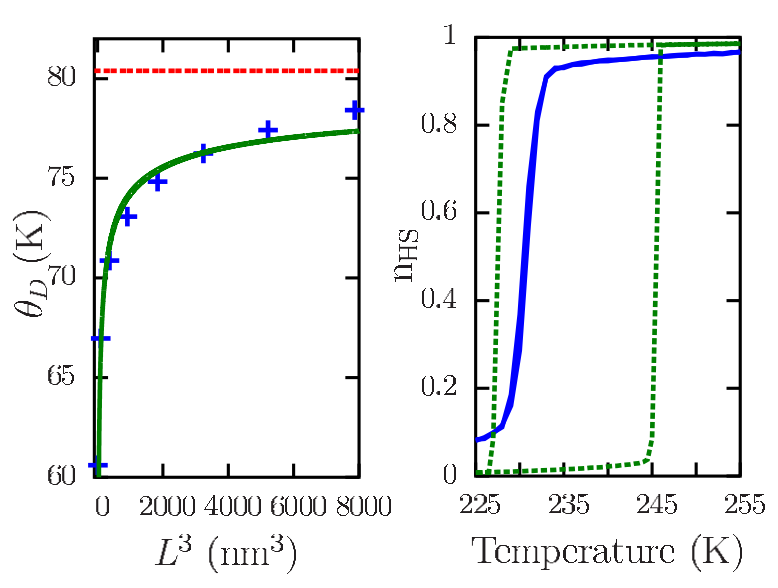

FIG. 3. (Color online) (a) Size dependence of the Debye temperature $\theta_{D}$ for a cubic lattice (blue crosses) with $\epsilon=6000 \mathrm{~K}$. For each size, $\theta_{D}$ has been determined from the MC estimation of $\left\langle u^{2}\right\rangle$ at a fixed temperature $T=200 \mathrm{~K}$. These data have been fitted by equation (10) linking $\theta_{D}$ and the melting temperature $T_{m}$ (green straight line). The dotted red line corresponds to the Debye temperature in the thermodynamic limit $\left(L \gg L_{c}\right)$. (b) Application to the spin transition: Monte Carlo simulation of the thermal evolution of the high spin fraction for a $N=4^{3}$ cubic system with free surfaces (blue straight line). The SCO properties of the corresponding bulk material (green dotted line) is also shown for comparison. The cohesion energies $A\left(\sigma_{i}, \sigma_{j}\right)$ (lattice parameters $\left.r_{\langle i, j\rangle}^{0}\right)$ are $A(1,1)=6100 \mathrm{~K}$ $\left(r_{\mathrm{HS}}=5.15 \AA\right), A(-1,-1)=6200 \mathrm{~K}\left(r_{\mathrm{LS}}=5.1 \AA\right)$, and $A(-1,1)=$ $A(1,-1)=6000 \mathrm{~K}\left(r_{\mathrm{HL}}=r_{\mathrm{LH}}=5.125 \AA\right)$ for the HS-HS, LS-LS, and HS-LS/LS-HS bonds, respectively. The coding heating rate is $4 \times 10^{-4} \mathrm{~K} \mathrm{MCS}^{-1}$.

surface effects get more and more importance and modify the vibrational properties of the whole system by changing the value of $\left\langle u_{i}\right\rangle$ initially present in the core. Therefore the mean-square displacement of the nanoparticles can be rather different from those of the corresponding bulk materials. This size dependence of lattice dynamics has a direct impact on the size evolution of the acoustic vibrational properties of the crystal which can be quantified by the size dependence of the Debye temperature as shown in Fig. 3(a). In the case of free boundary conditions and in the absence of a modification of the surface elastic properties, the nanoparticle apparently becomes "softer" than the corresponding bulk material and a decrease of the Debye temperature is observed. It is important to note that the relation linking the Debye temperature and the elastic properties of the materials is different at the nanometer scale than in the thermodynamic limit [32], so that the notion of "soft" and "hard" has to be carefully used.

It is interesting to establish a relation between the Debye $\theta_{D}$ and the melting $T_{m}$ temperatures of materials. Size effects in melting process have been studied for a long time and many similarities have been observed between solid-liquid and structural first-order transitions in nanocrystals [12], mainly due to the fact that these two characteristic temperatures $T_{m}$ and $\theta_{D}$ are both dependent on the mean-square displacement $\left\langle u^{2}\right\rangle$. An expression of the dependence with the size of the Debye temperature can be derived using a simple definition of the melting temperature and the Young-Laplace relation, which introduces the energetic cost for the creation of free surfaces [42] (see Appendix B):

$$
\theta_{D}=\sqrt{T_{m}^{b}-\alpha \frac{4 \gamma}{L}},
$$

where $T_{m}^{b}$ is the melting temperature for a bulk material. $\alpha$ and $\gamma$ correspond to a material dependent parameter and the surface energy, respectively. The numerical simulation in Fig. 3(a) has been fitted fairly well by Eq. (10) with a slight discrepancy mainly due to the fact that the surface energy $\gamma$ becomes itself size dependent for very small system sizes. Nevertheless, this simple analytical approach allows to establish a link between size dependencies of melting process and the lattice dynamics. Therefore a systematic study of the size dependence of the melting temperature of a material constitutes a new interesting way to predict the evolution of the vibrational properties with the size diminution.

However, the increase with the system size of the melting temperature [43] as well as of the Debye temperature [31,44] can occur under certain conditions. These have been attributed to (1) a higher sensibility to the environment (matrix effects) in which the nanoparticles are embedded in Ref. [28], (2) a modification of the surface mechanical properties due to chemical reactions or surface reconstruction, or (3) a hardening of the whole nanoparticle. Mössbauer spectroscopy measurements have recently highlighted a hardening of Prussian blue analog nanoparticles by approaching the nanoscale, which results in an increase of the Debye temperature [31]. This very important
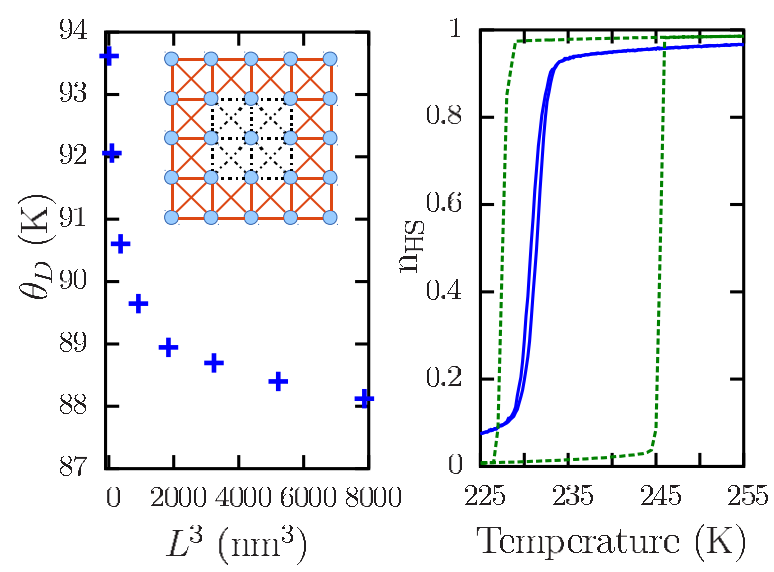

FIG. 4. (Color online) (a) Simulation of the size evolution of the Debye temperature $\theta_{D}$ for a core-shell nanoparticle where surface bond energies $\epsilon_{\text {surf }}=18000 \mathrm{~K}$ have been increased in comparison with the cohesion energy of the core, which is the same as in Fig. 3. (Insert) Schematic representation of the simulated core-shell system. (b) Application to the spin transition: Monte Carlo simulation of the thermal evolution of the high spin fraction for a core-shell cubic system of size $N=4^{3}$. The reopening of the hysteresis loop induced by the modification of surfaces is compared with the SC0 properties of the corresponding bulk material (green dotted line). Model and simulation parameters are the same as in Fig. 3 except the cohesion energies of HS-HS, LS-LS, and HS-LS (LS-HS) bonds at the surface, which have been increased $[A(1,1)=18100$ $\mathrm{K}, A(-1,-1)=18200 \mathrm{~K}$, and $A(-1,1)=A(1,-1)=18000 \mathrm{~K}]$, keeping the difference in stiffness between the HS and LS phases constant. 


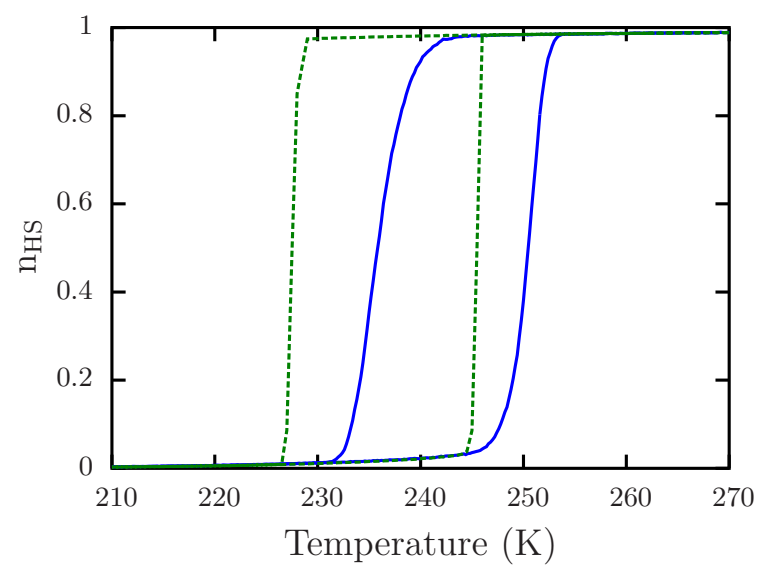

FIG. 5. (Color online) Monte Carlo simulation of the thermal evolution of the high spin fraction for a core-shell cubic system of size $N=4^{3}$ (blue straight line). The large reopening of the hysteresis loop is induced by an increase of the surface cohesion energy by making the assumption that the cohesion energy changes in a different way for the HS and LS states. The hysteresis loop of the nanoparticle is compared with the hysteresis loop of the corresponding bulk material (green dotted line). Model and simulation parameters are the same as in Fig. 4 except the cohesion energies of HS-HS, LS-LS, and HS-LS (LS-HS) bonds at the surface $[A(1,1)=18150 \mathrm{~K}, A(-1,-1)=18300 \mathrm{~K}$, and $A(-1,1)=A(1,-1)=18000 \mathrm{~K}]$.

result demonstrates that the decrease of the coordination number per site can be counterbalanced by an enhancement of the elasticity. The size evolution of the Debye temperature observed in Fig. 3(a) can be modified using specific boundary conditions, as for example, by increasing (ad hoc) the stiffness of the bonds at the surface, forming a core-shell structure [see Fig. 4(a)]. It is important to note that the surface/bulk vibrational coupling is dependent on the nature of the bonds linking the core and the shell of the nanoparticle, which is comparable with the role of coherent/semicoherent interfaces in the transmission of matrix elastic stresses to embedded nanoparticles [45].

The modulation of the elastic properties of nanoparticles by surface/interface effects has an impact on the control of the phase transition governed by electron-phonon couplings like the spin transition. Indeed, the consequences of these results on the SCO behavior at very small sizes can be simulated using Eq. (4). Starting from a same cooperative material, two extreme situations can be identified at the nanometric scale, according to the surface vibrational properties. In the case of free boundary conditions, the decrease of the Debye temperature of the nanoparticle leads to the loss of the bistability [Fig. 3(b)], whereas a small hysteresis loop occurs when the stiffness of the surface bonds is increased [Fig. 4(b)]. Although the hysteresis loop can be induced by kinetic effects in the case of small particle sizes, the transition curves of the two compared nanoparticles have been performed in the same numerical conditions (size, cooling/heating rates, etc.) and are also submitted to same kinetic effects. Hence, in the present work, the reopening of the hysteresis loop at small sizes has been induced by a hardening of the surface, which has caused a hardening of the whole nanoparticle due to the surface-volume vibrational coupling. In this approach, the difference in terms of stiffness $[A(1,1), A(-1,-1)$ and $A(1,-1)=A(-1,1)]$, known to play a major role in the existence of the bistability phenomenon [38], has been supposed to remain constant when the size is decreased. Size dependence of this difference would lead to the existence of huge shifts of the transition temperature, large hysteresis loop at the nanometer scale or change in phase stability as shown in Fig. 5. Moreover, this would strongly affect the nucleation energy barrier and cooperative mechanisms. Despite some observations of SCO properties at the nanoscale, experimental proofs for this behavior are still lacking.

\section{CONCLUSIONS}

In this paper, we show that the elastic constant of spinphonon models, in the SCO field, can be obtained from experimental measurements, allowing a more quantitative study and a more realistic simulation of the role of lattice deformations during the spin transition.

Furthermore, we show that the interface vibrational properties are not limited to the surface of the nanoobjects, but are strongly delocalized within the nanomaterials due to the long-range character of elastic interactions. The spatial distribution of the mean-square displacement allows for the definition of a characteristic thickness in which the surface vibrational properties cannot be neglected. When the particle size has the same order of magnitude as this characteristic thickness, the elastic properties of the whole nanoparticle are completely modified.

In particular, the mechanism of phase transitions governed by an electron-phonon phenomenon like the spin transition can be controlled by the surface stiffness. In the case of free surfaces, size reduction leads to a decrease of the Debye temperature and a loss of hysteretic behavior is observed for the nanoparticle. At the opposite side, when the surfaces are hardened by local structural reconstruction or by the external environment, the stiffness of the core-shell nanoparticle is increased and the bistability phenomenon persists even at very small sizes.

The origin of the surface hardening that modifies the elastic surface energy has still to be established. Even more important is to understand how the elastic interface energy can be affected by the external environment and how the surface elastic waves of the shell are coupled with the acoustic modes of the core. This can be achieved by the study of the core-shell nanoparticle vibrational spectra beyond the classical treatment of phonons.

Interestingly, the size dependence of the Debye temperature has been related to that of the melting temperature, which constitutes a new experimental way of comparison to better understand how the thermodynamic properties and kinetic processes during the spin transition can be modified by approaching the nanometer scale.

\section{ACKNOWLEDGMENTS}

The authors are grateful for the financial support from the project ANR Nanohybrid (ANR-13-BS07-0020-01). This work is supported by Labex Numev and EADS Fundation. 


\section{APPENDIX A: EXTRACTION OF A CHARACTERISTIC SURFACE THICKNESS $\lambda$}

The strong variation of the local mean-square displacement by approaching the surfaces of the nanoparticles can be characterized by a distance $\lambda$ defining a depth in which the surface and the core are strongly coupled.

This distance $\lambda$ can be deduced from an exponential fit of the profile of $\left\langle u_{i}^{2}\right\rangle$ by analogy with finite size ferromagnetic systems whose energy is modeled by the Ising Hamiltonian exhibiting surface effects. Indeed, it has been analytically demonstrated that the spontaneous magnetization density exhibits an exponential decay by approaching a free surface [46]. We note that the profile of $\left\langle u_{i}^{2}\right\rangle$ cannot be perfectly fitted by an exponential law mainly due to the fact that the local mean-squared displacement is directly related to the elastic properties of the crystal packing, which corresponds to long-range interactions as opposed to the short-range character of the exchange coupling in the Ising model. Therefore the exponential fit systematically underestimates $\lambda$.

From this value, we can deduce the surface "thickness" as $5 \lambda$ and the critical size of the nanoparticle $L_{c}=10 \lambda \approx$ $7-8 \mathrm{~nm}$ below which surface properties are predominant. The characteristic thickness $\lambda$ is virtually independent on the system size. Obviously, below $L_{c}$, the extracted value of the distance $\lambda$ becomes more and more meaningless since the effects of the different surfaces of the cubic system overlap, which drastically modifies the vibrational properties of the nanoparticle. In other words, below $L_{c}$, the vibrational properties of the nanoparticle are dominated by surface effects.

\section{APPENDIX B: SIMPLE ANALYTICAL APPROACH FOR THE SIZE DEPENDENCE OF THE MELTING AND THE DEBYE TEMPERATURES}

In the harmonic limit and in the framework of the Debye model, the melting temperature $T_{m}$ can be expressed as $[47,48]$

$$
T_{m} \propto \frac{c_{L}^{2}\left(r_{\langle i j\rangle}^{0}\right)^{2} M \omega_{D}^{2}}{k_{B}},
$$

where $M$ and $\omega_{D}=k_{B} \theta_{D} / \hbar$ are the mass and the Debye angular momentum of the bonds $\langle i j\rangle . c_{L}$ is the so-called Lindmann criterion, which is a semiempirical estimation of the melting temperature and can be related to the mean-square displacement by

$$
c_{L}=\sqrt{\frac{\left\langle u^{2}\right\rangle}{3\left(r_{\langle i j\rangle}^{0}\right)^{2}}} .
$$

The solid is considered to melt for an arbitrary value of the Lindmann criterion $\left(c_{L} \approx 10 \%-14 \%\right)$. A dependence of the melting temperature with the system size can be established using thermodynamic considerations and the well-known Young-Laplace relation:

$$
T_{m}=T_{m}^{b}-\alpha \frac{4 \gamma}{L}
$$

where $\alpha$ is a material dependent parameter and $\gamma$ is the surface energy. By injecting Eq. (B3) in Eq. (B1), an expression of the dependence with the size of the Debye temperature can be derived:

$$
\theta_{D} \propto \sqrt{T_{m}^{b}-\alpha \frac{4 \gamma}{L}} .
$$

[1] A. Bousseksou, G. Molnár, L. Salmon, and W. Nicolazzi, Chem. Soc. Rev. 40, 3313 (2011).

[2] M. Cavallini, Phys. Chem. Chem. Phys. 14, 11867 (2012).

[3] H. J. Shepherd, G. Molnár, W. Nicolazzi, L. Salmon, and A. Bousseksou, Eur. J. Inorg. Chem. 2013, 653 (2013).

[4] G. Molnár, L. Salmon, W. Nicolazzi, F. Terki, and A. Bousseksou, J. Mater. Chem. C. 2, 1360 (2014).

[5] Spin Crossover in Transition Metal Compounds, Topics in Current Chemistry Vol. 233-235, edited by P. Gütlich and H. A. Goodwin (Springer, Berlin, Heidelberg, 2004).

[6] V. Ksenofontov, G. Levchenko, H. Spiering, P. Gütlich, J.-F. Letard, Yacine Bouhedja, and O. Kahn, Chem. Phys. Lett. 294, 545 (1998).

[7] S. Bonhommeau, G. Molnár, M. Goiran, K. Boukheddaden, and A. Bousseksou, Phys. Rev. B 74, 064424 (2006).

[8] S. Decurtins, P. Gütlich, C. P. Köhler, H. Spiering, and A. Hauser, Chem. Phys. Lett. 105, 1 (1984).

[9] S. Bedoui, M. Lopes, W. Nicolazzi, S. Bonnet, S. Zheng, G. Molnár, and A. Bousseksou, Phys. Rev. Lett. 109, 135702 (2012).

[10] H. Spiering, K. Boukheddaden, J. Linares, and F. Varret, Phys. Rev. B. 70, 184106 (2004).

[11] J. A. Nasser, Eur. Phys. J. B 21, 3 (2001).
[12] M. Mikolasek, G. Félix, W. Nicolazzi, G. Molnár, L. Salmon, and A. Bousseksou, New. J. Chem. 38, 1834 (2014).

[13] I. Boldog, A. B. Gaspar, V. Martínez, P. Pardo-Ibañez, V. Ksenofontov, A. Bhattacharjee, and P. Gütlich, and J. A. Real, Angew Chem. 47, 6433 (2008).

[14] F. Volatron, L. Catala, E. Rivieère, A. Gloter, O. Steéphan, and T. Mallah, Inorg. Chem. 47, 6584 (2008).

[15] T. Forestier, A. Kaiba, S. Pechev, D. Denux, P. Guionneau, C. Etrillard, N. Daro, E. Freysz, and J.-F. Létard, Chem. Eur. J. 15, 6122 (2009).

[16] A. Rotaru, F. Varret, A. Gindulescu, J. Linares, A. Stancu, J. F. Létard, T. Forestier, and C. Etrillard, Eur. Phys. J. B 84, 439 (2011).

[17] J. Galán-Mascarós, E. Coronado, A. Forment-Aliaga, M. Monrabal-Capilla, E. Pinilla-Cienfuegos, and M. Ceolin, Inorg. Chem. 49, 5706 (2010).

[18] L. Larionova, L. Salmon, Y. Guari, A. Tokarev, K. Molvinger, G. Molnár, and A. Bousseksou, Angew. Chem. 47, 8236 (2008).

[19] I. A. Guralskiy, C. M. Quintero, G. Molnár, I. O. Fritsky, L. Salmon, and A. Bousseksou, Chem. Eur. J. 18, 9946 (2012).

[20] Y. Raza, F. Volatron, S. Moldovan, O. Ersen, V. Huc, C. Martini, F. Brisset, A. Gloter, O. Stephan, A. Bousseksou, L. Catala, and T. Mallah, Chem. Comm. 47, 11501 (2011). 
[21] P. Durand, S. Pillet, E.-E. Bendeif, C. Carteret, M. Bouazaoui, H. el Hamzaoui, B. Capoen, L. Salmon, S. Hebert, J. Ghanbaja, L. Arandah, and D. Schaniel, J. Mat. Chem. C 1, 1933 (2013).

[22] A. Tissot, C. Enachescu, and M.-L. Boillot, J. Mater. Chem. 22, 20451 (2012).

[23] T. Kawamoto and S. Abe, Chem. Commun., 3933 (2005).

[24] A. Muraoka, K. Boukheddaden, J. Linarès, and F. Varret, Phys. Rev. B 84, 054119 (2011).

[25] D. Chiruta, J. Linares, P. R. Dahoo, and M. Dimian, Physica B: Condens. Matter 435, 76 (2014).

[26] Daniel Chiruta, Catalin-Maricel Jureschi, Jorge Linares, Yann Garcia, and Aurelian Rotaru, J. Appl. Phys. 115, 053523 (2014).

[27] Alexandru Atitoaie, Radu Tanasa, and Cristian Enachescu, J. Magn. Magn. Mater. 324, 1596 (2012).

[28] H. Oubouchou, A. Slimani, and K. Boukheddaden, Phys. Rev. B 87, 104104 (2013).

[29] L. Stoleriu, P. Chakraborty, A. Hauser, A. Stancu, and C. Enachescu, Phys. Rev. B 84, 134102 (2011).

[30] C. Brechignac, P. Houdy, and M. Lahmani, Nanomaterials and Nanochemistry (Springer, Berlin, 2007).

[31] G. Félix, W. Nicolazzi, L. Salmon, G. Molnár, M. Perrier, G. Maurin, J. Larionova, J. Long, Y. Guari, and A. Bousseksou, Phys. Rev. Lett. 110, 235701 (2013).

[32] G. Félix, W. Nicolazzi, M. Mikolasek, G. Molnár, and A. Bousseksou, Phys. Chem. Chem. Phys. 16, 7358 (2014).

[33] W. Nicolazzi, S. Pillet, and C. Lecomte, Phys. Rev. B 78, 174401 (2008).

[34] A. Bousseksou, J. Nasser, J. Linares, K. Boukheddaden, and F. Varret, J. Phys. I 2, 1381 (1992).
[35] W. Nicolazzi and S. Pillet, Phys. Rev. B. 85, 094101 (2012).

[36] A. Slimani, K. Boukheddaden, F. Varret, H. Oubouchou, M. Nishino, and S. Miyashita, Phys. Rev. B 87, 014111 (2013).

[37] J. A. Nasser, S. Topçu, L. Chassagne, M. Wakim, B. Benali, J. Linares, and Y. Alayli, Eur. Phys. J. B 83, 115 (2011).

[38] W. Nicolazzi, J. Pavlik, S. Bedoui, G. Molnár, and A. Bousseksou, Eur. Phys. J. Spec. Top. 222, 1137 (2013).

[39] N. Metropolis, A. W. Rosenbluth, M. N. Rosenbluth, A. H. Teller, and E. Teller, J. Chem. Phys. 21, 1087 (1953).

[40] N. N. Greenwood and T. C. Gibb, Mössbauer Spectroscopy (Chapman and Hall, London, 1971).

[41] R. L. McGreevy, J. Phys.: Condens. Matter 13, R877 (2001).

[42] P. Letellier, A. Mayaffre, and M. Turmine, Phys. Rev. B. 76, 045428 (2007).

[43] Q. Jiang, Z. Zhang, and J. C. Li, Chem. Phys. Lett. 322, 549 (2000).

[44] M. X. Gu, Chang Q. Sun, Z. Chen, T. C. Au Yeung, S. Li, C. M. Tan, and V. Nosik, Phys. Rev. B 75, 125403 (2007).

[45] K. Lu and Z. H. Jin, Curr. Opin. Solid State Mater. Sci. 5, 39 (2001).

[46] K. Binder, Critical Behaviour at Surfaces, edited by C. Domb and J. L. Lebowitz, Phase Transitions and Critical Phenomena Vol. 8 (Academic Press, London, 1983), pp. 145-266.

[47] C. C. Yang, M. X. Xiao, W. Li, and Q. Jiang, Solid State Commun. 139, 148 (2006).

[48] G. Ouyang, Z. M. Zhu, W. G. Zhu, and C. Q. Sun, J. Phys. Chem. C 114, 1805 (2010). 